\title{
Two cases of dual origin left vertebral arteries with non-convergence
}

\author{
Kimberly A Congdon, Nicole Clifton, Jennifer Wuellner, Laurie Stapleton
}

Congdon KA, Clifton N, Wuellner J, et al. Two cases of dual origin left vertebral arteries with non-convergence. Int J Anat Var. 2019; 12(4): 52-54.

In typical human anatomy, the vertebral arteries (VA) originate from the subclavian arteries (SCA) and enter the transverse foramen of the sixth cervical vertebra (C6). The reported frequency of deviations vary, but the clinical significance of a more medial location of aberrant LVAs result in a need to understand their frequency in the population, as well as any coexisting vascular variations. Here we reported 2 cases of variant left vertebral arteries that entered the transverse foramen of the $\mathrm{C} 4$ vertebra and continued to the brain. Both presented with a second vessel entering the C6 transverse foramen, suggesting a dual origin of the left vertebral artery, but in neither case did the two vessels converge. These findings suggest a higher frequency of occurrence of this anatomical variation, and demonstrate the continued importance of cadaver-based anatomical study.

Key Words: Vertebral artery; Neck anatomy; Cadaver study; Cardiovascular variation

\section{INTRODUCTION}

$\mathrm{I}^{\mathrm{n}}$ typical human anatomy, the vertebral arteries (VA) originate from the subclavian arteries (SCA), enter the transverse foramen of the sixth cervical vertebra (C6), and travel within the subsequent more superior transverse foramina to enter the skull via the foramen magnum, and provide circulation to the posterior brain. Reported frequencies of VAs with aberrant origins vary widely, and have been reported in anywhere from 5 to $50 \%$ of the population [1-3]. Of those, left vertebral artery (LVA) variants are most common, appearing in approximately 2 to $7 \%$ of the population (as reported in a recent aggregation of studies) [1]. Approximately $90 \%$ of those are part of an atypical aortic arch (AA) branching pattern [3]. The clinical significance of a more medial location of these aberrant LVAs [1,3-6], in conjunction with their oft-observed more superior entry into the $\mathrm{C} 4$ transverse foramen (rather than C6) [7] result in a need to understand their frequency in the population, as well as any co-existing vascular variations. Dual origin VAs are even less common [1]. They have been reported in less than 50 individuals $[1,8]$ with varying frequency of right side, left side, or bilateral presentations. Dual origin VAs present additional clinical concerns, as they have been previously misdiagnosed as a dissection or fenestration of the VA [5]. They have also been reported to be indicators of increased risk of dissection, and have anecdotally been linked to pain, dizziness, headache, nausea, cognitive impairment, and other pathologies [1,3]. Additionally, many reports of aberrant LVAs document a more superior transverse foramen entry than normal $[1,7]$ but they do not explicitly discuss the presence of arteries in the more inferior foramina (which would indicate a dual origin VA), so this phenomenon may be underreported. Here, we describe two cases of dual origin left vertebral arteries.

\section{CASE REPORT}

During the course of normal anatomical dissection practices as part of the osteopathic medicine program's human gross anatomy course at Touro University Nevada, one cadaver was found to have an aberrant LVA originating from the AA rather than the SCA. Following this discovery, all bodies were surveyed and a second cadaver with a similar aberrant LVA was discovered. Upon further dissection, it was found that both cadavers presented with dual origin vessels, one that entered the C6 transverse foramen and one that entered the $\mathrm{C} 4$ transverse foramen. In neither case was convergence between the two vessels observed. The entire sample consisted of 38 adult cadavers, 20 females and 18 males. Ages ranged from 38 to 100, with a mean age of 78 . Causes of death and other health information were reported by the individual or donating family member. No other demographic data was available on the donors. Bodies were supplied by the University of Texas Southwestern Body Donor Program.

\section{RESULTS}

Case 1: Male, 93, cause of death stated as senile degeneration of the brain The LVA originated from the AA, distal and posterior to the left common carotid artery (CCA) and proximal to the left SCA. It entered the transverse foramen of the $\mathrm{C} 4$ vertebra and continued to the brain. A single branch arising from the left subclavian artery proximal to the thyrocervical trunk entered the transverse foramen of the C6 vertebra and continued through the transverse foramen of the $\mathrm{C} 5$ vertebra, ending as branches serving the C4 vertebra (Figure 1).

Case 2: Female, 79, cause of death stated as COPD/respiratory failure. The LVA originated from the AA, distal and slightly posterior to the left CCA, proximal to the left subclavian artery. It entered the transverse foramen of the $\mathrm{C} 4$ vertebra and continued to the brain. Three independent branches arising from the left subclavian artery proximal to the thyrocervical trunk converged into one vessel, entered the transverse foramen of the C6 vertebra, and continued through the transverse foramen of the $\mathrm{C} 5$ vertebra, ending as branches serving the $\mathrm{C} 4$ vertebra (Figure 2).

\section{DISCUSSION}

The presence of a dual origin vertebral artery is an embryological variation in development caused by the persistence of metameric arteries [9]. Metameric arteries supply the central and peripheral nervous systems, the dura mater, and the bone, muscles and skin that correspond to their segmental level. In normal development, the vertebral artery forms from a longitudinal anastomosis of metameric arteries. When considering the dual origin VA, the level of entrance into the transverse foramina has been suggested to

Department of Anatomy, Basic Sciences Faculty, Nevada College of Osteopathic Medicine, Touro University, 874 American Pacific Drive, Henderson, NV 89014, USA

Correspondence: Dr. Kimberly A Congdon, Department of Anatomy, Basic Sciences Faculty, Nevada College of Osteopathic Medicine, Touro University 874 American Pacific Drive, Henderson, NV 89014, USA. Telephone+702-777-4771; E-mail: Kimberly.congdon@tun.touro.edu

Received: Jul 23, 2019, Accepted: Oct 29, 2019, Published: Nov 04, 2019 org/licenses/by-nc/4.0/), which permits reuse, distribution and reproduction of the article, provided that the original work is properly cited and the reuse is restricted to noncommercial purposes. For commercial reuse, contact reprints@pulsus.com 


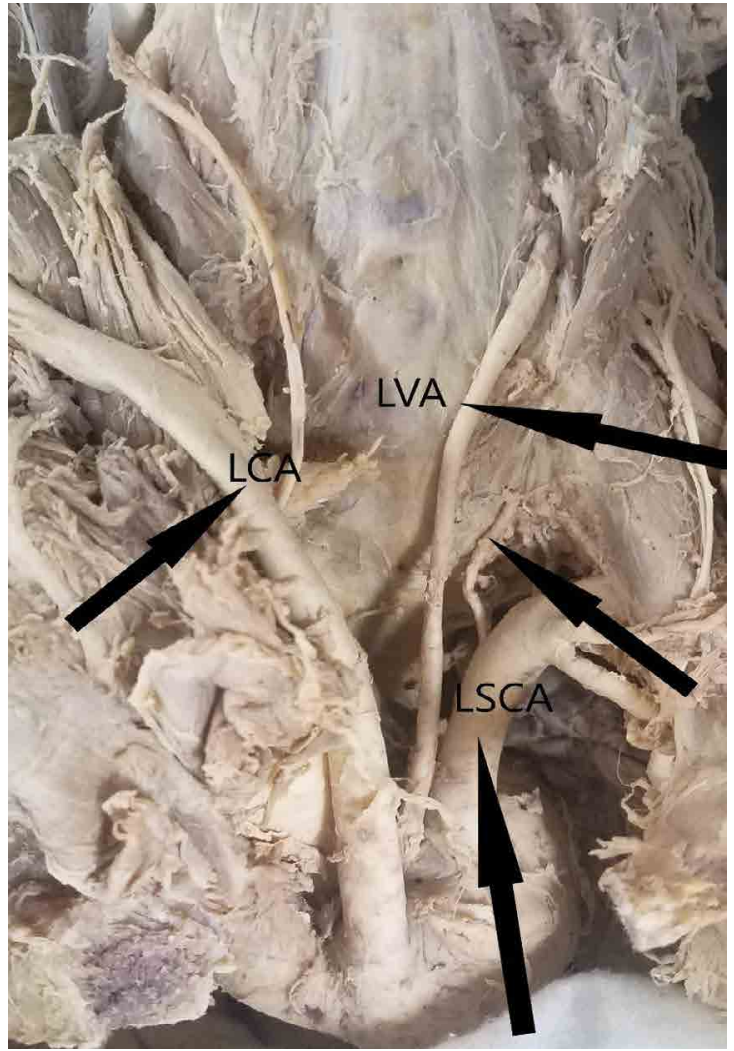

Figure 1) Case Study 1. LCA: Left Common Carotid Artery; LSCA: Left Subclavian Artery; LVA: Left Vertebral Artery; DOA: Dual Origin Artery. Note the LVA originating from the aortic arch medial to the LSCA and the DOA originating from the LSCA directly.

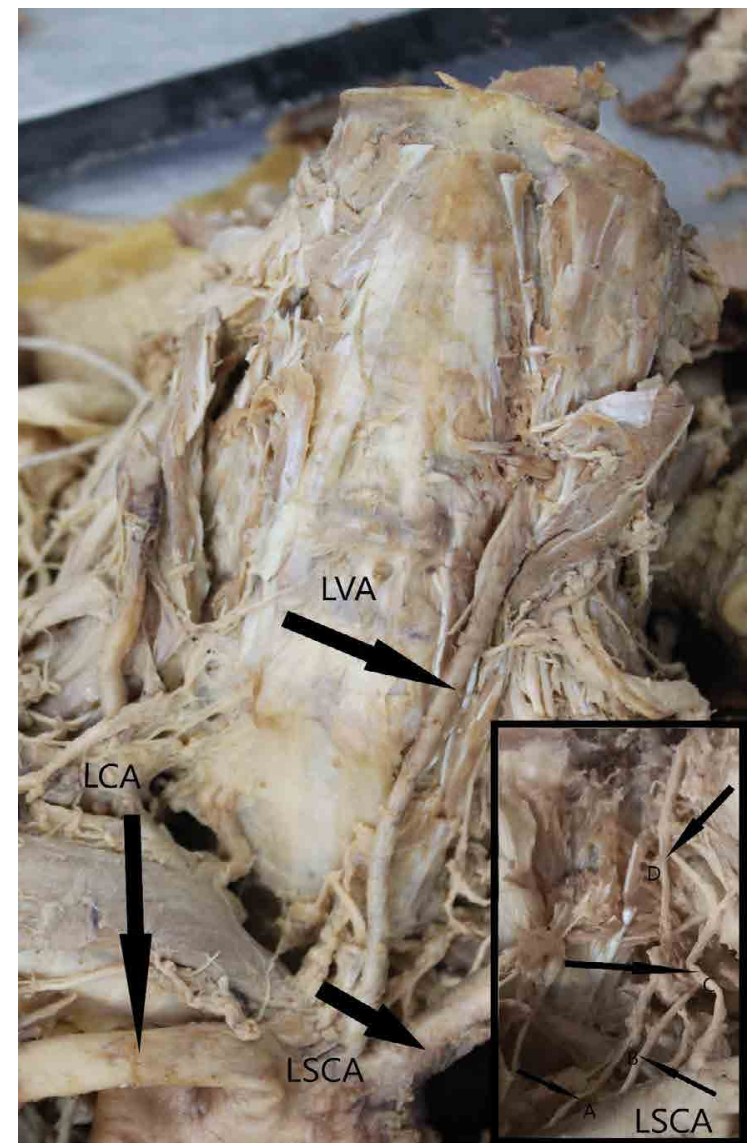

Figure 2) Case Study 2. LCA: Left Common Carotid Artery, LSCA: Left Subclavian Artery, LVA: Left Vertebral Artery, DOA: Dual Origin Artery. Insert shows close-up of vessels formIng DOA (A, B and C) originating from LSCA and anastomosing at D to form DOA. correspond with the persistent metameric artery in question [9]. Therefore, for both of our cases, it appears that a normal VA began to develop from the SCA and entered the C6 transverse foramen, and the ascending cervical artery originating from the $\mathrm{C} 3-\mathrm{C} 4$ metamere entered the $\mathrm{C} 4$ transverse foramen and persisted throughout embryological development into adulthood. Thus, the vessel serving the brain is the persistent metameric vessel and the typically-developing VA is identified as the dual origin variant. However, unlike most reported cases (but see) [10], we could not identify a joining of the two vessels. It appears that the VA of SCA origin ends as branches serving the $\mathrm{C} 5$ and $\mathrm{C} 4$ vertebrae just proximal to the entrance of the vessel of AA origin in both cases.

Finding two dual origin vertebral arteries in a sample of 38 individuals suggests a much higher frequency (approximately $5 \%$ of the total population) than has been previously reported $[3,11]$ and would increase the number of reported cases by roughly $4 \%$. This suggests the frequency of dual origin vertebral arteries is underreported. Aberrant vertebral arteries have been associated with headaches and vertigo [4]. They have been associated with a higher risk of arterial dissection [6,9] and can complicate numerous surgical approaches, particularly any anterior approaches to the spine $[1,3,11]$ although most reports and publications on this variant consider it largely non-pathological $[3,4]$. The relatively benign nature of this anatomical variation may be contributing to an underestimated frequency. However, the fact that this variant appears to be more frequent in females than males [2] and as sex-based differences in pain perception by medical professionals has been recorded [12] we must consider the possibility that this variant underlies conditions that are being overlooked by health care providers. Improving our understanding of the frequency of this variant and educating health care practitioners to its possible presence could improve the quality of health care for many people. Additionally, understanding the range of variation, frequency of occurrence, and common associated variations or pathologies is necessary to improving surgical outcomes and assisting radiologists and neurologists in diagnosis.

\section{CONCLUSION}

The first of the two cases described here were discovered by a medical student during routine dissection for the head and neck unit in the cadaver lab of the first year gross anatomy course, near the end of the academic year. Had this student not been knowledgeable enough to identify the aberrant presentation, it might not have come to the attention of faculty. It was only as a result of this first discovery that we proceeded to survey all the other bodies and discovered a second presentation that had been previously overlooked. When considering the uncertainty that remains regarding the range of nonpathological anatomical variation and the rates of occurrence of variants linked to pathological conditions in the population, we are struck by the risk that is run by medical schools and other human anatomy-focused programs transitioning away from cadaver-based dissection and towards digital replacements. There was nothing in the medical history of either of the individuals described here to suggest they had any knowledge of their variant vertebral artery (but see for a discussion of a possible causal link to cerebral disorders regarding Case Study 1). Had they not been generous enough to donate their remains, these variations would have gone unrecorded, as would the majority of what we know about human anatomical variation. Beyond the value of cadaver-based dissection to anatomical learning, access to human cadavers is crucial to our understanding of the true range of human anatomical variation. We hope this fact will be taken into account in the ongoing discussion of the role of digital replacements in anatomy education.

\section{ACKNOWLEDGEMENTS}

We would like to acknowledge the assistance of anatomy lab manager Jeremy Day. We would also like to acknowledge the generous donation of these two cadavers, and all individuals, families and loved ones who make body donations to educational programs like ours.

\section{REFERENCES}

1. Woraputtaporn $\mathrm{W}$, Ananteerakul T, Iamsaard S, et al. Incidence of vertebral artery of aortic arch origin, its level of entry into transverse foramen, length, diameter and clinical significance. Anat Sci Int 2019;94:275-79.

2. Einstein EH, Song LH, Villela NLA, et al. Anomalous Origin of the Left Vertebral Artery from the Aortic Arch. Aorta. 2016;4:64-7. 
3. Lazaridis N, Piagkou M, Loukas M, et al. A systematic classification of the vertebral artery variable origin: clinical and surgical implications. Surg Radiol Anat. 2018;40:779-97.

4. Kim MS. Duplicated Vertebral Artery: Literature Review and Clinical Significance. J Korean Neurosurg Soc. 2018;61:28-34.

5. Nogueira TE, Chambers AA, Brueggemeyer MT, et al. Dual Origin of the Vertebral Artery Mimicking Dissection. AJNR Am J Neuroradiol. 1996; 18:382-4.

6. Yuan SM. Aberrant Origin of Vertebral Artery and its Clinical Implications. Braz J Cardiovasc Surg. 2016;31:52-9.

7. Vujmilovic S, Spasojevic G, Vujnovic S, et al. Variability of the vertebral artery origin and transverse foramen entrance level - CT angiographic study. Folia Morphol. 2018;77:687-92.
8. Kritikos M, Sharma N, Sedora-Roman N, et al. Duplication of the vertebral artery: A series of four cases. Interv Neuroradiol. 2018;24:596600.

9. Meila D, Tysiac M, Petersen M, et al. Origin and Course of the Extracranial Vertebral Artery: CTA Findings and Embryologic Considerations. Clin Neuroradiol. 2012;22:327-33.

10. Watanabe K, Saga T, Iwanaga J, et al. A rare case of dual origin of the left vertebral artery without convergence. Folia Morphol. 2016;75:136-42.

11. Baik J, Baek HJ, Shin HS, et al. Duplication of the right vertebral artery: MRA findings and review of the literature. Springer Plus. 2016;5:1123-6.

12. Hoffman DE, Tarzian AJ. The Girl Who Cried Pain: A Bias Against Women in the Treatment of Pain. J Law Med Ethics. 2001;29:13-27. 